\title{
The impact of labor induction at 39 weeks gestation compared with expectant management on maternal and neonatal morbidity in low-risk women: A United States of America Cohort Study
}

\author{
Sabrina Burn ${ }^{1}$, Ruofan Yao ${ }^{2}$, Maria Diaz ${ }^{2}, J_{o r d a n}$ Rossi $^{2}$, and Stephen Contag ${ }^{1}$ \\ ${ }^{1}$ University of Minnesota \\ ${ }^{2}$ Loma Linda University
}

December 28, 2020

\begin{abstract}
Objective: To determine maternal and neonatal morbidity associated with induction of labor at 39 weeks compared with expectant management through 42 weeks. Design: Cohort study Setting \& Population: Low risk American women who delivered between 39 and 42 weeks in 2015 to 2017. Methods: Data was abstracted from the national vital statistics database. Multivariable log-binomial regression analysis was conducted to estimate the relative risk of morbidity. Main Outcome Measures: Maternal morbidity included Triple I, blood transfusion, ICU admission, uterine rupture, cesarean hysterectomy, and cesarean delivery. Neonatal morbidity included 5 minute Apgar [?]3, prolonged ventilation, seizures, NICU admission, and neonatal death. Results: A total of 1,885,694 women were included for analysis. Women undergoing induction of labor at 39 weeks were less likely to develop Triple I (p-value $<0.001$; aRR 0.66; 95\% CI [0.64-0.68]) and require a cesarean section (p-value $<0.01$; aRR 0.691 95\% CI [0.68-0.69]) than the expectant management group. There was a small, but significant increase in cesarean hysterectomy in the induction group (p-value $<0.01$; aRR 1.32; 95\% CI [1.05-1.65]). Neonates of the induction group were less likely to have 5 minute Apgar [?]3 (p-value < 0.01; aRR 0.69; 95\% CI [0.64-0.74]), prolonged ventilation (p-value < 0.01; aRR $0.77 ; 95 \%$ CI [0.72-0.82]), NICU admission (p-value < 0.01; aRR 0.80; 95\% CI [0.79-0.82]), and/or neonatal seizures (p-value $<0.01$; aRR 0.80; 95\% CI [0.66-0.98]) compared to the expectant management group. Conclusions: Induction of labor at 39 weeks gestation compared with expectant management is not harmful and has maternal and neonatal benefits.
\end{abstract}

The impact of labor induction at 39 weeks gestation compared with expectant management on maternal and neonatal morbidity in low-risk women: A United States of America Cohort Study

\section{Short title}

39 week induction of labor: a United States of America cohort study

Sabrina C. Burn, $M D^{*}{ }^{1}$, Ruofan Yao, $M D, M P H^{2}$, Maria Diaz, $M D^{2}$, Jordan Rossi, $M D^{2}$ and Stephen Contag, $M D^{1}$

${ }^{1}$ Department of Obstetrics, Gynecology \& Women's Health, University of Minnesota, Minneapolis, MN, USA

${ }^{2}$ Department of Obstetrics \& Gynecology, Loma Linda University School of Medicine, Loma Linda, CA, USA

\section{Disclosure}

The author(s) report no conflict of interest 


\section{Funding}

This research received no specific grant from any funding agency in the public, commercial or not-for-profit sectors.

\section{Presentation}

Presented at the Society for Maternal-Fetal Medicine's 40th Annual Meeting,

February 3-8, 2020, Grapevine, Texas

\section{Corresponding author}

Sabrina C. Burn, MD

Department of Obstetrics, Gynecology \& Women's Health, University of Minnesota,

420 Delaware Street, SE, MMC 395, Minneapolis, MN, USA 55455

Phone: (317) 345-3653

Email: burn@umn.edu

\section{Abstract}

Objective: To determine maternal and neonatal morbidity associated with induction of labor at 39 weeks compared with expectant management through 42 weeks.

Design : Cohort study

Setting \& Population : Low risk American women who delivered between 39 and 42 weeks in 2015 to 2017.

Methods: Data was abstracted from the national vital statistics database. Multivariable log-binomial regression analysis was conducted to estimate the relative risk of morbidity.

Main Outcome Measures: Maternal morbidity included Triple I, blood transfusion, ICU admission, uterine rupture, cesarean hysterectomy, and cesarean delivery. Neonatal morbidity included 5 minute Apgar [?]3, prolonged ventilation, seizures, NICU admission, and neonatal death.

Results: A total of 1,885,694 women were included for analysis. Women undergoing induction of labor at 39 weeks were less likely to develop Triple I (p-value $<0.001$; aRR 0.66; 95\% CI [0.64-0.68]) and require a cesarean section (p-value <0.01; aRR $0.69195 \%$ CI [0.68-0.69]) than the expectant management group. There was a small, but significant increase in cesarean hysterectomy in the induction group (p-value $<0.01$; aRR 1.32; 95\% CI [1.05-1.65]). Neonates of the induction group were less likely to have 5 minute Apgar [?]3 (p-value < 0.01; aRR 0.69; 95\% CI [0.64-0.74]), prolonged ventilation (p-value $<0.01 ;$ aRR 0.77; 95\% CI [0.72-0.82]), NICU admission (p-value < 0.01; aRR 0.80; 95\% CI [0.79-0.82]), and/or neonatal seizures (p-value <0.01; aRR 0.80; 95\% CI [0.66-0.98]) compared to the expectant management group.

Conclusions: Induction of labor at 39 weeks gestation compared with expectant management is not harmful and has maternal and neonatal benefits.

Tweetable abstract: Induction of labor at 39 weeks is associated with both maternal and neonatal benefits.

Keyword s: Induction of labor, expectant management, 39 weeks gestation, maternal morbidity, neonatal morbidity

\section{Introduction}

Decisions regarding expectant management and timing of induction of labor must take into consideration both maternal and neonatal risks. Traditionally, it was believed that induction of labor prior to 41 weeks gestation is associated with increased maternal and neonatal risks. ${ }^{1,2}$ More specifically, several observational and retrospective cohort studies found that women who underwent induction of labor prior to 41 weeks 
gestation had an increased frequency of maternal and neonatal morbidity when compared to women who went into spontaneous labor at similar gestational ages. ${ }^{1-8}$ Thus, as a standard in obstetrical clinical management elective induction of labor in low-risk women was generally avoided before 41 weeks gestation because of a lack of neonatal benefits and adverse maternal outcomes.

Recent research has challenged this standard and current guidelines for low-risk women by more appropriately comparing the maternal, perinatal, and neonatal consequences of induction of labor at 39 weeks to those managed expectantly. ${ }^{9-21}$ Most of these studies have not shown a higher risk for adverse outcomes with labor induction at 39 weeks, except in cases of trial of labor after cesarean delivery. ${ }^{22}$ Some have shown that induction of labor resulted in more favorable maternal and perinatal outcomes than expectant management. ${ }^{15-19}$ However, many of these studies were very limited in sample size, studied specific age groups and were not powered to detect significant maternal and neonatal morbidities. ${ }^{15-19}$ Similarly, the results of the randomized landmark studies, including the Walker et al. and the ARRIVE trial, were not completely consistent and didn't provide definitive evidence of any potential advantages or disadvantages of labor induction at 39 weeks versus expectant management outside of a clinical trial. ${ }^{20,21}$ It is with these previously published findings in mind, that we designed the current national, large-scale retrospective cohort study

\section{Methods}

This study is a national retrospective cohort analysis using data abstracted from the National Center for Health Statistics (NCHS) and Centers for Disease Control and Prevention's (CDC) Division of Vital Statistics database from 2015 to 2017. We chose the most recent 3 years of available data to be feasibly analyzed with our available computing hardware limits. The data is publicly available and de-identified, therefore institutional review board approval was not required.

Pregnancies with delivery $<39$ weeks and $0 / 7$ days gestation or $>42$ weeks and $6 / 7$ days gestation, multifetal gestation, fetal congenital anomalies, pregestational diabetes, gestational diabetes, chronic hypertension, previous cesarean delivery $(\mathrm{CD})$, and infant deaths were excluded from the study cohort. Infant deaths, defined as death that occurred after 28 days of life, were excluded due to its association with postnatal complications often unrelated to the birth process. ${ }^{23,24}$ Deliveries $>42$ weeks and $6 / 7$ days gestation were excluded primarily because it is no longer common practice to continue expectant management at this gestational age given the inherent neonatal morbidities associated with post-date delivery. ${ }^{25,26}$ Common maternal comorbidities were excluded to isolate the effects of elective induction of labor at 39 weeks compared with expectant management in an otherwise low-risk population. In addition to the above exclusions, the induction of labor group excluded all pregnancies diagnosed with gestational hypertensive disorders, as the American College of Obstetricians and Gynecologist (ACOG) recommends delivery for such disorders on or after 37 weeks gestation. ${ }^{27}$ As such, expectant management would not be a reasonable option in these situations. Lastly, those with spontaneous deliveries at 39 weeks of gestation who did not undergo induction of labor were also excluded.

The induction of labor group consisted of deliveries by induction of labor between 39 weeks and $0 / 7$ days to $6 / 7$ days gestation without an identifiable medical indication, irrespective of their final mode of delivery. The expectant management group consisted of all spontaneous deliveries from 40 weeks and $0 / 7$ days through 42 weeks and 6/7 days gestation. Maternal demographic information was compared between the two management groups using the appropriate univariate statistical test. Statistical significance was defined as p-value $<0.01$.

The maternal outcomes of interest included: cesarean delivery, intra-amniotic infection or inflammation (triple I), blood transfusion, intensive care unit (ICU) admission, uterine rupture, and cesarean hysterectomy. Triple I, or chorioamnionitis as it was previously known, was identified as "clinical chorioamnionitis diagnosed during labor or maternal temperature greater than or equal to 38 (100.4)." The neonatal outcomes of interest included: 5-minute Apgar score [?]3, assisted ventilation for $>6$ hours, neonatal intensive care unit (NICU) admission, seizure, and neonatal death (death before 28 days of life). This data was collected 
through the first 28 days of life. Both maternal and neonatal data from these databases were obtained from birth certificate data. Multivariable log-binomial regression analysis was performed to control for potential confounding variables based on historic significance and univariable analysis. These variables included: maternal age, race, parity, education, prenatal care, tobacco use, and body mass index (BMI). Backward stepwise elimination method was performed to arrive at the final regression model, which included maternal age, race, education, and vaginal delivery. Power analysis was not performed as this study's sample size included the entire population. All analyses were performed using Stata 14 statistical software (College Station, TX). ${ }^{28}$

\section{Results}

There were 5,984,942 pregnancies available for review from the NCHS and CDC's Division of Vital Statistics database from January 1st, 2015 to December 31st, 2017. The data from a total of 1,885,694 singleton, non-anomalous pregnancies that met inclusion criteria were abstracted from the database. Of these pregnancies, 424,690(22.5\%) women underwent induction of labor at 39 weeks gestation, while the remaining $1,461,004(77.5 \%)$ women delivered spontaneously after expectant management through 42 weeks and $0 / 7$ days gestation (Figure 1 ).

Maternal demographics and clinical characteristics were similar between the two groups and are summarized in Table 1. In both groups, the mean maternal age was 28.0 years; the most common race was White followed by Black; the majority of women had a high school diploma or above in terms of education; and the mean body mass index (BMI) was 26.7 in the induction of labor group and 26.0 in the expectant management group. Approximately half of the women in each group had a prior vaginal delivery.

Maternal outcomes were analyzed for both the induction of labor and the expectant management group. The results are summarized in Table 2. Maternal morbidity was evaluated by comparing the frequency of triple I, blood transfusion, ICU admission, uterine rupture, cesarean hysterectomy, and cesarean delivery between the two groups. All results were adjusted for obesity, education, race and prior vaginal delivery. Mothers were significantly less likely to be diagnosed with triple I in the induction of labor group when compared to the expectant management group (p-value < 0.001; aRR: 0.66; 95\% CI [0.64-0.68]) and had a decreased rate of cesarean section (p-value <0.01; aRR $0.69195 \%$ CI [0.68-0.69]) in the expectant management group. The cesarean hysterectomy rate was approximately 2 per 10,000 deliveries for the entire cohort. Women who underwent induction of labor were over $30 \%$ more likely to have undergone a cesarean hysterectomy (p-value <0.01; aRR 1.32; 95\% CI [1.05-1.65]). Conversely, there were no differences between the two groups in maternal frequency of blood transfusion, ICU admission, or uterine rupture.

Neonatal outcomes were analyzed for both the induction of labor and the expectant management group. A summary of the results can be found in Table 3. Neonatal morbidity was evaluated by comparing the risk of neonates with 5 min Apgar [?]3, prolonged ventilation, NICU admission, seizure, and neonatal death between the two groups. All results were adjusted for obesity, education, race and prior vaginal delivery. Neonates in the induction group were significantly less likely to have: (i) 5 min Apgar [?] 3 (p-value $<0.01$; aRR 0.69; 95\% CI [0.64-0.74]), (ii) require prolonged ventilation (p-value $<0.01$; aRR 0.77 ; $95 \%$ CI $[0.72$ $0.82]$ ), (iii) necessitate NICU admission (p-value < 0.01; aRR 0.80; 95\% CI [0.79-0.82), and/or (iv) neonatal seizures (p-value $<0.01$; aRR 0.80; 95\% CI [0.66-0.98]) when compared to the expectant management group. Conversely, there was no difference in the frequency of neonatal death between the two groups.

\section{Discussion}

\section{Main Findings}

This national retrospective cohort study analyzed data from a total of 1,885,694 singleton, non-anomalous, low-risk pregnancies in the United States of America. From a maternal perspective, our data demonstrates a significantly lower frequency of triple I and cesarean delivery in women who underwent induction of labor at 39 weeks gestation as compared to the expectant management group. Induction of labor was associated with increased rates of cesarean hysterectomy. Although the risk was found to be $30 \%$ higher than the expectant 
management group, it is important to note that the absolute risk for this complication remains very low and was detected due to this study's large sample size. Labor induction did not result in any statistically significant difference in the frequency of blood transfusion, ICU admission, or uterine rupture when the two groups were compared. From a neonatal perspective, our data demonstrates that labor induction at 39 weeks gestation resulted in a significantly lower frequency of 5 minute Apgar [?] 3, requirement for ventilation, seizures and/or NICU admission than in the expectant management group. In contrast, labor induction did not result in any significant difference in the frequency of neonatal death between the two groups.

Taken together, these findings demonstrate that induction of labor at 39 weeks gestation is associated with: (i) a lower risk of developing triple I and requiring a cesarean delivery, (ii) a small increased risk for cesarean hysterectomy, and (iii) a significantly reduced frequency of neonatal morbidity when compared with an expectantly managed pregnancy.

\section{Strengths $\mathscr{E}$ Limitations}

The strength of the current study is the large and heterogeneous sample size of pregnancies analyzed in both the induction of labor at 39 weeks gestation group and the expectant management group. An additional strength of the study is the data quality. The data collected and analyzed is current, reliable, and includes data from the entirety of the United States of America. Lastly, the study was adjusted for significant confounders including, obesity, education, race and prior vaginal delivery. Limitations of the study include the inability to review indication for induction of labor, methods utilized for induction of labor, bishop scores, fetal monitoring, severity of bleeding requiring transfusion, severity of uterine rupture versus dehiscence, and/or indications for intervention such as cesarean delivery. However, it is assumed that there is enough similarity in practice as recommended by ACOG that this would not directly impact the data collected. In addition, there is no possibility of verifying the veracity of the data obtained. Previous evaluation of the validity of this data supports it is reliable and has a high degree of completeness and accuracy. ${ }^{29}$ If misclassifications occurs, they are likely to be secondary to under reporting rather than over reporting. This therefore would lead to higher baseline risk in the control population and therefore an underestimation of the risk in the study population.

\section{Interpretation}

Two of the previously published landmark studies regarding induction of labor at 39 weeks gestation versus expectant management were the randomized trials by Walker et al. and the ARRIVE trial. ${ }^{20}$, 21 The Walker et al. trial compared labor induction at 39 weeks gestation with expectant management in women 35 years of age or older. ${ }^{20}$ The primary outcome of the study was to determine the rate of cesarean delivery in each group. Secondary outcomes evaluated maternal and neonatal morbidity and serious neonatal complications. Their results indicated that induction of labor at 39 weeks did not result in a significant difference in adverse short-term effects on maternal or neonatal outcomes, including cesarean section. In contrast our study demonstrated that induction of labor at 39 weeks gestation resulted in a significantly lower risk of maternal triple I, cesarean section, and neonatal morbidity as compared to expectant management through 42 weeks. This difference may be explained by the fact that the Walker et al. study was relatively small and homogeneous sample size (619 subjects), looked only at women of 35 years of age or older, and was powered to primarily test the hypothesis that induction of labor would reduce the rate of cesarean section. In contrast our cohort study with the much larger sample size of 1,885,694 subjects including women of all ages may have allowed detecting between-group differences of individual maternal and neonatal morbidities.

The ARRIVE trial was designed to test the hypothesis that elective induction of labor at 39 weeks would result in a lower risk of a composite outcome of perinatal death or severe neonatal complications than expectant management among low-risk nulliparous women. ${ }^{21}$ Their results indicated that induction of labor at 39 weeks did not result in a significantly lower frequency of a composite adverse perinatal outcome, but it did result in a significantly lower frequency of cesarean delivery. Consistent with the data, our study demonstrates there is a significantly lower rate of cesarean section in the induction group compared to the expectant-management group. In contrast to the ARRIVE trail, however, our study shows a significantly 
lower frequency of adverse neonatal outcomes between the induction of labor and the expectant management group. These differences may be explained by the fact that the ARRIVE trial took place in a solely academic center and therefore may have had a relatively homogeneous subject population. In addition, despite its sample size, the trial was not powered to detect differences in rare adverse outcomes.

A novel finding of our study is the increased rate of cesarean hysterectomy in the induction of labor group. This new finding was identified due to the large study population, which is powered to detect rare adverse outcomes such as this. The increased rate of cesarean hysterectomy cannot be explained exclusively by differences in the rates of uterine rupture or postpartum hemorrhage, the two most common indications. Previous research has demonstrated that induction of labor and the methods used for induction of labor are associated with an increased risk for uterine rupture. Induction agents, such as prostaglandins and oxytocin, have been associated with risk for uterine atony and rupture, which are the two most common indications to proceed with cesarean hysterectomy. ${ }^{30-34}$ Due to the nature of this study, the indication for cesarean hysterectomy could not be determined in this cohort, however, the overall rate is consistent with previous rates seen with induction of labor. It is likely that a combination of these factors led to an increased rate of cesarean hysterectomy among women undergoing induction of labor. With this new finding in mind, it is important to note that induction of labor is not without consequence.

\section{Conclusions}

In conclusion, our study demonstrates that in low-risk women induction of labor at 39 weeks gestation is not associated with any adverse effects on maternal or neonatal outcomes, but it is significantly associated with both lower frequencies of maternal and neonatal morbidity when compared to expectant management through 42 weeks. Thus, avoiding or delaying the induction of labor at or after 39 weeks gestation may not always be in the best interest of the mother nor the neonate. Based on our study and supported by a growing body of literature a careful discussion of current policies and a reconsideration of current standard clinical protocols aimed at the avoidance of induction of labor at 39 weeks gestation in low-risk women seems warranted.

Acknowledgements : We would like to thank the National Center for Health Statistics (NCHS) and Centers for Disease Control and Prevention's (CDC) Division of Vital Statistics for the creation and maintenance of this freely accessible database.

Disclosures of interests: No conflicts of interest to declare.

Contribution to Authorship: SC and RY planned the study and obtained the necessary data. All authors (SCB, RY, MD, JR, SC) contributed to the analysis of the data and interpretation of results. SCB drafted the manuscript and all other authors assisted with editing of the manuscript. All authors have accepted it in its final form.

Details of Ethics Approval: This study is a national retrospective cohort analysis using data abstracted from the NCHS and CDC's Division of Vital Statistics database. The data is publicly available and de-identified, therefore no patients were directly involved and no ethical approval was required.

Funding : None

\section{References}

1. Macer JA, Macer CL, Chan LS. Elective induction versus spontaneous labor: a retrospective study of complications and outcomes. Am J Obstet Gynecol. 1992 Jun;166:1690-6.

2. Maslow AS, Sweeny AL. Elective induction of labor as a risk factor for cesarean delivery among low-risk women at term. Obstet Gynecol.2000 Jun;95(6 Pt 1):917-22.

3. Cammu H, Martens G, Ruyssinck G, et al. Outcome after elective labor induction in nulliparous women: a matched cohort study. Am J Obstet Gynecol. 2002 Feb;186(2):240-4.

4. Luthy DA, Malmgren JA, Zingheim RW. Cesarean delivery after elective induction in nulliparous women: the physician effect. Am J Obstet Gynecol. 2004 Nov;191(5):1511-5. 
5. Vrouenraets FP, Roumen FJ, Dehing CJ, van den Akker ESA, Aarts MJB, Scheve EJT. Bishop score and risk of cesarean delivery after induction of labor in nulliparous women. Obstet Gynecol. 2005 Apr;105(4):690-7.

6. Dunne C, Da Silva O, Schmidt G, Natale R. Outcomes of elective labour induction and elective cesarean section in low-risk pregnancies between 37 and 41 weeks' gestation. J Obstet Gynaecol Can. 2009 Dec;31(12):1124-30.

7. Ehrenthal DB, Jiang X, Strobino DM. Labor induction and the risk of a cesarean delivery among nulliparous women at term. Obstet Gynecol. 2010 Jul;116(1):35-42.

8. Vardo JH, Thornburg LL, Glantz JC. Maternal and neonatal morbidity among nulliparous women undergoing elective induction of labor.J Reprod Med. 2011 Jan-Feb;56(1-2):25-30.

9. Osmundson S, Ou-Yang RJ, Grobman WA. Elective induction compared with expectant management in nulliparous women with an unfavorable cervix. Obstet Gynecol. 2011 Mar;117(3):583-7.

10. Stock SJ, Ferguson E, Duffy A, Ford I, Chalmers J, Norman JE. Outcomes of elective induction of labour compared with expectant management: population based study. BMJ. 2012 May 10;344:e2838.

11. Cheng YW, Kaimal AJ, Snowden JM, Nicholson JM, Caughey AB. Induction of labor compared to expectant management in low risk women and associated perinatal outcomes. Am J Obstet Gynecol. 2012 Dec;207(6):502e1-8.

12. Darney BG, Snowden JM, Cheng YW, Jacob L, Nicholson JM, Kaimal A, et al. elective induction of labor at term compared with expectant management: maternal and neonatal outcomes. Obstet Gynecol.2013 Oct;122:761-9.

13. Mishanina E, Rogozinska E, Thatthi T, Uddin-Khan R, Khan KS, Meads C. Use of labour induction and risk of cesarean delivery: a systematic review and meta-analysis. CMAJ. 2014 Jun 10;186(9):66573.

14. Gibson KS, Waters TP, Bailit JL. Maternal and neonatal outcomes in electively induced low-risk term pregnancies. Am J Obstet Gynecol. 2014 Sep;211(3):249.e1-16.

15. Middleton P, Shepherd E, Crowther CA. Induction of labour for improving birth outcomes for women at or beyond term. Cochrane Database Syst Rev. 2018 May 9;5(5): CD004945.

16. Grobman WA, Caughey AB. Elective induction of labor at 39 weeks compared with expectant management: a meta-analysis of cohort studies.Am J Obstet Gynecol. 2019 Oct;221(4):304-10.

17. Saccone G, Della Corte L, Maruotti GM. Induction of labor at full-term in pregnant women with uncomplicated singleton pregnancy: A systematic review and meta-analysis of randomized trials. Acta Obstet Gynecol Scand. 2019 Aug;98(8):958-66.

18. Sotiriadis A, Petousis S, Thilaganathan B, Figueras F, Martins WP, Odibo AO, et al. Maternal and perinatal outcomes after elective induction of labor at 39 weeks in uncomplicated singleton pregnancy: a meta-analysis. Ultrasound Obstet Gynecol. 2019 Jan;53(1):26-35.

19. Society of Maternal-Fetal Medicine (SMFM) Publications Committee. SMFM Statement on Elective Induction of Labor in Low-Risk Nulliparous Women at Term: the ARRIVE Trial. Am J Obstet Gynecol. 2019 Jul;221(1):B2-4.

20. Walker KF, Bugg GJ, Macpherson M, McCormick C, Grace N, Wildsmith C, et al. Randomized trial of labor induction in women 35 years of age or older. $N$ Engl $J$ Med. 2016 Mar;374:813-22.

21. Grobman WA, Rice MM, Reddy UM, Tita ATN, Silver RM, Mallett G, et al; Eunice Kennedy Shriver National Institute of Child Health and Human Development Maternal-Fetal Medicine Units Network. Labor induction versus expectant management in low-risk nulliparous women. $N$ Engl J Med. 2018 Aug 9;379(6):513-23.

22. Park BY, Cryer A, Betoni J, McLean L, Figueroa H, Contag SA, Yao R. Outcomes of labor induction at 39 weeks in pregnancies with a prior cesarean delivery. J Matern Fetal Med 2020 Aug 26:1-6.

23. Matthews TJ, MacDorman MF, Thoma ME. Infant mortality statistics from 2013 period linked birth/infant death data set. Natl Vital Stat Rep. 2015 Aug 6;64(9):1-30.

24. Goldstein RD, Trachtenberg FL, Sens MA, Harty BJ, Kinney HC. Overall postneonatal mortality and rates of SIDS. Pediatrics. 2016 Jan;137(1).

25. Tunón K, Eik-Nes SH, Grøttum P. Fetal outcome in pregnancies defined as post-term according to 
the last menstrual period estimate, but not according to the ultrasound estimate. Ultrasound Obstet Gynecol. 1999 Jul;14(1):12-16.

26. Clausson B, Cnattingius S, Axelsson O. Outcomes of post-term births: the role of fetal growth restriction and malformations. Obstet Gynecol. 1999 Nov;94(5 Pt 1):758-62.

27. American College of Obstetricians and Gynecologists. Hypertension in pregnancy: report of the American College of Obstetricians and Gynecologists' Task Force on Hypertension in Pregnancy. Obstet Gynecol. 2013 Nov;122:1122-31.

28. StataCorp. 2015. Stata Statistical Software: Release 14. College Station, Tx: StataCorp LP.

29. Gregory ECW, Martin JA, Argov EL, Osterman MJK. Assessing the quality of medical and health data from the 2003 birth certificate revision: results from new york city. National Vital Statistics Reports 2019 Jun;68(8):1-20.

30. Grotegut CA, Paglia MJ, Johnson LNC, Thames B, James AH. Oxytocin exposure during labor among women with postpartum hemorrhage secondary to uterine atony. Am J Obstet Gynecol. 2011 Jan;204(1):56.e1-6.

31. Wetta LA, Szychowski JM, Seals S, Mancuso MS, Biggio JR, Tita ATN. Risk factors for uterine atony/postpartum hemorrhage requiring treatment after vaginal delivery. Am J Obstet Gynecol.2013;209(1):51.e1-6.

32. Balki M, Ramachandran N, Lee S, Talati C. The recovery time of myometrial responsiveness after oxytocin-induced desensitization in human myometrium in vitro. Anesth Analg. 2016 May;122(5):150815.

33. Al-Zirqi I, Daltveit AK, Forsen L, Stray-Pedersen B, Vangen S. Risk factors for complete uterine rupture. Am J Obstet Gynecol. 2017 Feb;216:165.e1-8.

34. Thisted DLA, Mortensen LH, Krebs L. Uterine rupture without previous cesarean delivery: a population-based cohort study. Eur J Obstet Gynecol Reprod Biol. 2015 Dec;195:151-5.

\section{Hosted file}

BJOG_Tables.pdf available at https://authorea.com/users/385745/articles/500965-the-impactof-labor-induction-at-39-weeks-gestation-compared-with-expectant-management-on-maternaland-neonatal-morbidity-in-low-risk-women-a-united-states-of-america-cohort-study

\section{Hosted file}

BJOG_Figure.pdf available at https://authorea.com/users/385745/articles/500965-the-impactof-labor-induction-at-39-weeks-gestation-compared-with-expectant-management-on-maternaland-neonatal-morbidity-in-low-risk-women-a-united-states-of-america-cohort-study 